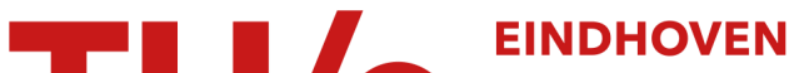 \\ UNIVERSITY OF \\ TECHNOLOGY
}

\section{Separation of simultaneous rain and ice depolarization}

Citation for published version (APA):

Kamp, van de, M. M. J. L. (2004). Separation of simultaneous rain and ice depolarization. IEEE Transactions on Vehicular Technology, 52(2), 513-523. https://doi.org/10.1109/TAP.2003.818776

DOI:

10.1109/TAP.2003.818776

Document status and date:

Published: 01/01/2004

Document Version:

Publisher's PDF, also known as Version of Record (includes final page, issue and volume numbers)

Please check the document version of this publication:

- A submitted manuscript is the version of the article upon submission and before peer-review. There can be important differences between the submitted version and the official published version of record. People interested in the research are advised to contact the author for the final version of the publication, or visit the $\mathrm{DOI}$ to the publisher's website.

- The final author version and the galley proof are versions of the publication after peer review.

- The final published version features the final layout of the paper including the volume, issue and page numbers.

Link to publication

\section{General rights}

Copyright and moral rights for the publications made accessible in the public portal are retained by the authors and/or other copyright owners and it is a condition of accessing publications that users recognise and abide by the legal requirements associated with these rights.

- Users may download and print one copy of any publication from the public portal for the purpose of private study or research.

- You may not further distribute the material or use it for any profit-making activity or commercial gain

- You may freely distribute the URL identifying the publication in the public portal.

If the publication is distributed under the terms of Article $25 \mathrm{fa}$ of the Dutch Copyright Act, indicated by the "Taverne" license above, please follow below link for the End User Agreement:

www.tue.nl/taverne

Take down policy

If you believe that this document breaches copyright please contact us at:

openaccess@tue.nl

providing details and we will investigate your claim. 


\title{
Separation of Simultaneous Rain and Ice Depolarization
}

\author{
Max M. J. L. van de Kamp
}

\begin{abstract}
In the case of depolarization due to rain or to ice crystals, the differential attenuation, the differential phase shift, and the canting angle of the depolarizing medium can all be calculated independently from dual polarized measurement results. However, this is not possible when rain and ice are causing depolarization at the same time. This paper presents a calculation method which makes it possible to derive during simultaneous rain and ice depolarization some of the characterising parameters of each part separately. The method makes use of an a priori known relation between the differential attenuation and the differential phase shift of rain and of ice. The calculation method is tested on measured depolarization events, and yields good results.
\end{abstract}

Index Terms-Ice, millimeter wave propagation, nonhomogeneous media, polarization, rain.

\section{INTRODUCTION}

$\mathbf{R}$ AINSTORMS and high-altitude ice clouds cause depolarization on satellite links. This effect has been modeled by various researchers, e.g., [1]-[8]. Rain causes depolarization of radio waves by anisotropic attenuation and phase shift. The typical raindrop has an approximate spheroidal shape, which has, looking in the direction of propagation, two orthogonal symmetry axes (Fig. 1), as long as the elevation angle of the link is less than $90^{\circ}$. The eccentricity of the spheroid increases with drop size. The orientation of the symmetry axes is influenced by wind sheer forces [1], [2].

The orientations of all raindrops in a rainstorm are distributed around a mean orientation, which gives the medium two orthogonal axes of symmetry when seen in the direction of propagation. These symmetry axes are canted with respect to the horizontal and the vertical over a canting angle, generally equal to the mean raindrop canting angle. The rainstorm causes a different attenuation and phase shift for radio waves polarized along these two symmetry axes.

For arbitrary orthogonal polarizations $x$ and $y$, the result is crosstalk between the two channels, called depolarization. The measured parameters of depolarization are the cross polarization discrimination XPD, which is the ratio between received copolar and crosspolar signal levels; and the phase between coand crosspolar signals.

Several prediction models of depolarization due to rain and ice crystals have already been developed, e.g., [3]. This develop-

Manuscript received January 12, 2000; revised December 18, 2002.

The author was with ONERA/DEMR, 31055 Toulouse CEDEX 4, France. He is now with the Department of Electrical and Electronic Engineering, University of Bath, Bath, BA2 7AY, U.K.

Digital Object Identifier 10.1109/TAP.2003.818776

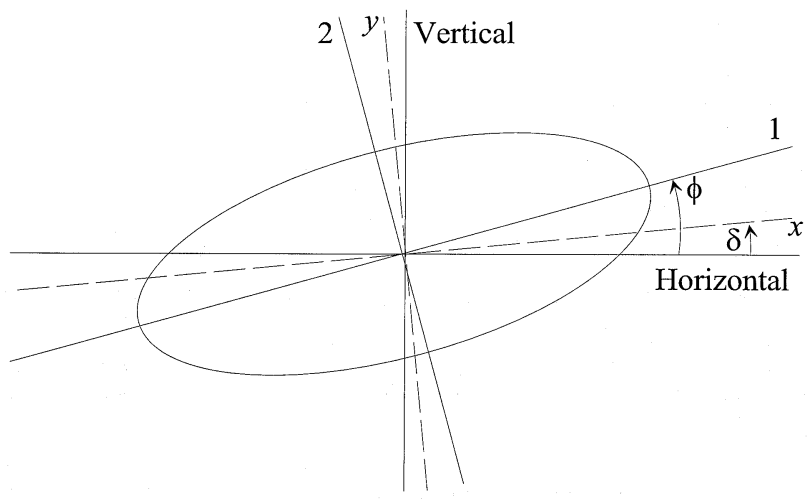

Fig. 1. Canting of a raindrop, seen in the polarization plane (i.e., perpendicular to the propagation direction). " 1 " and " 2 " are the symmetry axes of the raindrop; $x$ and $y$ are the polarization directions of the wave; "horizontal" is the intersection of the horizontal plane with the polarization plane; "vertical" is the projection of the vertical axis on the polarization plane. $\phi$ is the canting angle of the raindrop; $\delta$ is the polarization angle.

ment is being much facilitated by the identification of the characterising parameters of depolarising media and their relation to the measured XPD, and by the assessment of these parameters from measurements.

The characterising parameters of a depolarizing rainstorm are the differential attenuation, the differential phase shift, and the canting angle of the symmetry axes. In propagation experiments with a dual polarized signal, all these characterizing parameters can be calculated from the measured signals [4]-[6]. The differential attenuation and differential phase shift of rain appear to be to some extent correlated.

The other possible cause of depolarization is ice crystals, present in higher atmospheric layers [7], [8]. These hydrometeors have very high eccentricity, and may be aligned by electrostatic forces in thunderstorms. The ice medium generally has two axes of symmetry, similarly as in the case of rain. The same characterizing parameters can be identified as for rain. The main difference with the effect of raindrops is that ice crystals do not cause any significant attenuation and therefore no differential attenuation.

Rain and ice crystals can also cause depolarization at the same time. The occurrence of this has been observed by, e.g., Dintelmann et al. [9], who measured the differential attenuation and phase shift between the two polarizations of the Olympus $20 \mathrm{GHz}$ beacon. They found mostly either a relation which agreed with rain depolarization models, or a large differential phase shift without any significant differential attenuation. Sometimes however, they found an unusually large differential 


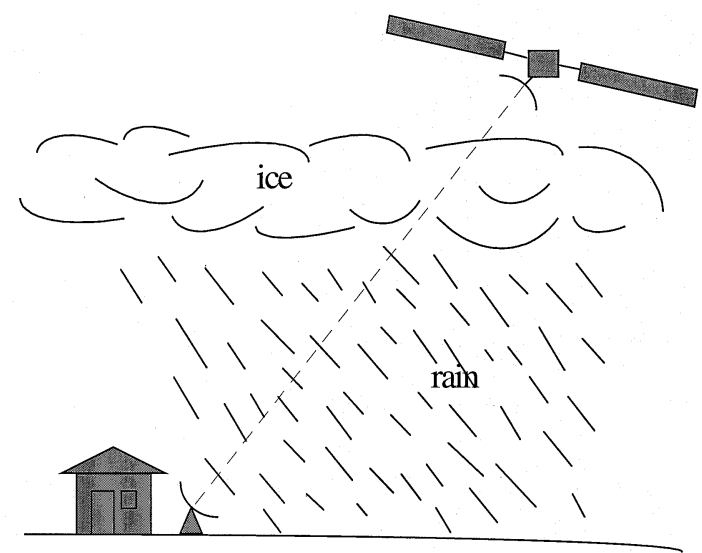

Fig. 2. Configuration with a double depolarizing medium.

phase shift during an otherwise "normal" behavior at high attenuation. They concluded that in these cases rain and ice must have been on the path at the same time.

In the case of simultaneous depolarization by rain and by ice, the assumption of a whole medium with two symmetry axes is no longer valid, since generally the two media do not have the same axes of symmetry. As a consequence, the anisotropy and canting angle are not unambiguously defined, and can not as easily be calculated anymore. However, in order to improve the prediction models of depolarization due to rain and ice crystals, it will be necessary to identify and determine the characterizing parameters also of such a double depolarizing medium. This paper shows a model defining such parameters, and a procedure using which these parameters can be calculated from measurements of simultaneous depolarization due to rain and to ice.

\section{Model of a Cascaded Depolarizing Medium}

A solution to the problem of calculating the characterizing parameters of a double depolarizing medium is found by using the assumption that the two parts are spatially separated. It may be expected that ice crystals usually are present in a higher atmospheric layer than a rainstorm. Because of this, the medium as a whole can be seen as a cascade of two different media (see Fig. 2). This can be expressed in the well-known matrix representation of the medium. In this representation, the received field is expressed as a vector of the two received orthogonal field components, and resulting from the transmitted field vector and a transmission matrix. This transmission matrix can now be written as a cascade of two matrices

$$
\begin{aligned}
{\left[\begin{array}{l}
E_{x} \\
E_{y}
\end{array}\right] } & =\left[\begin{array}{ll}
\tau_{x x} & \tau_{x y} \\
\tau_{y x} & \tau_{y y}
\end{array}\right]\left[\begin{array}{l}
E_{x} \\
E_{y}
\end{array}\right]_{t r} \\
& =\left[\begin{array}{ll}
r_{x x} & r_{x y} \\
r_{y x} & r_{y y}
\end{array}\right]\left[\begin{array}{ll}
i_{x x} & i_{x y} \\
i_{y x} & i_{y y}
\end{array}\right]\left[\begin{array}{l}
E_{x} \\
E_{y}
\end{array}\right]_{t r}
\end{aligned}
$$

with $t r$ indicating the transmitted field, and $r$ and $i$ denoting the transmission coefficients of rain and ice, respectively. The two matrices of the partial media can be described similarly as the theory for a single medium. Supposing the two partial media are homogeneous, the antidiagonal terms of the partial matrices are equal, as follows from the calculation model of a single medium e.g. [2], [6], [7]. Then the total matrix can be written as

$$
\begin{aligned}
{\left[\begin{array}{ll}
\tau_{x x} & \tau_{x y} \\
\tau_{y x} & \tau_{y y}
\end{array}\right] } & =r_{x y} i_{x y}\left[\begin{array}{cc}
X_{r} & 1 \\
1 & Y_{r}
\end{array}\right]\left[\begin{array}{cc}
X_{i} & 1 \\
1 & Y_{i}
\end{array}\right] \\
& =r_{x y} i_{x y}\left[\begin{array}{cc}
X_{r} X_{i}+1 & X_{r}+Y_{i} \\
X_{i}+Y_{r} & 1+Y_{r} Y_{i}
\end{array}\right]
\end{aligned}
$$

where $X_{r}$ and $Y_{r}$ are given by

$$
\begin{gathered}
X_{r}=\frac{r_{x x}}{r_{y x}}=\frac{\Gamma_{r}+\tan ^{2}\left(\phi_{r}-\delta\right)}{\left(\Gamma_{r}-1\right) \tan \left(\phi_{r}-\delta\right)} \\
Y_{r}=\frac{r_{y y}}{r_{x y}}=\frac{1+\Gamma_{r} \tan ^{2}\left(\phi_{r}-\delta\right)}{\left(\Gamma_{r}-1\right) \tan \left(\phi_{r}-\delta\right)}
\end{gathered}
$$

where $\phi_{r}-\delta$ is the canting angle of symmetry axis 1 of the rain medium relative to the polarization direction " $x$ " ( $\delta$ is the polarization angle). The (complex) parameter $\Gamma_{r}$ is given by

$$
\Gamma_{r}=\mathrm{e}^{\Delta_{r}}=\mathrm{e}^{-\left(\alpha_{r} \frac{\ln 10}{20}+j \beta_{r} \frac{\pi}{180}\right)}
$$

with $\alpha_{r}=$ differential attenuation $(\mathrm{dB}) ; \beta_{r}=$ differential phase shift $\left(^{\circ}\right) . \alpha_{r}$ and $\beta_{r}$ indicate the difference in attenuation and phase shift between signals polarized in the two symmetry axes of the medium. The parameter $\Delta_{r}$ is often named the "anisotropy" of the rain medium e.g. [5], [10], [11]. Similarly as in (3a), (3b), and (4), $X_{i}$ and $Y_{i}$ can be expressed in $\phi_{i}, \alpha_{i}$, and $\beta_{i}$.

The transmission matrix can be expressed in measured quantities as follows:

$$
\left[\begin{array}{ll}
\tau_{x x} & \tau_{x y} \\
\tau_{y x} & \tau_{y y}
\end{array}\right]=\tau_{x y}\left[\begin{array}{cc}
R Y & 1 \\
\frac{R Y}{X} & Y
\end{array}\right]
$$

with $R=\tau_{x x} / \tau_{y y}=$ the (complex) ratio between the received copolar signal levels; $X=\tau_{x x} / \tau_{y x}$ and $Y=\tau_{y y} / \tau_{x y}$ are the depolarization ratios of the entire medium.

These are related to measured quantities as

$$
\begin{aligned}
& \mathrm{XPD}_{x}=20 \log |X| \quad(\mathrm{dB}) \quad \psi_{x}=\arg X \\
& \mathrm{XPD}_{y}=20 \log |Y| \quad(\mathrm{dB}) \quad \psi_{y}=\arg Y \\
& R C P L=20 \log |R| \quad(\mathrm{dB}) \quad \psi_{r}=\arg R
\end{aligned}
$$

with $\mathrm{XPD}=$ the difference in $\mathrm{dB}$ between received copolar and crosspolar amplitudes of a signal transmitted at one polarization. $\mathrm{XPD}_{x, y}=\mathrm{XPD}$ of a signal transmitted at $x$ - and $y$-polarization, respectively; $\psi_{x, y}=$ phase between co- and crosspolar components of a signal. RCPL $=$ relative copolar level in $\mathrm{dB}$ between signals at $x$ - and $y$-polarizations; $\psi_{r}=$ phase between copolar signals at $x$ - and $y$-polarizations.

Combining (2) and (5), it follows that

$$
\left\{\begin{array}{l}
X=\frac{\left(X_{r} X_{i}+1\right)}{\left(X_{i}+Y_{r}\right)} \\
Y=\frac{\left(1+Y_{r} Y_{i}\right)}{\left(X_{r}+Y_{i}\right)} \\
R=\frac{\left(X_{r} X_{i}+1\right)}{\left(1+Y_{r} Y_{i}\right)}
\end{array}\right.
$$


After measuring $R, Y$, and $X$, the left-hand side of (7) is completely known. This is a system of three complex equations, or six real equations, with the unknowns on the right-hand side. Substituting (3a), (3b), and (4) into $X_{r}, Y_{r}, X_{i}$ and $Y_{i}$, it follows that the unknown variables are

- the canting angles $\phi_{r}$ and $\phi_{i}$ of the rain and ice media;

- the anisotropy parameters $\alpha_{r}, \alpha_{i}, \beta_{r}$ and $\beta_{i}$ of the rain and ice media.

If the equations formed by (7) are mutually independent, this system of six equations with six unknowns can be solved entirely and all of the parameter values can be determined.

\section{SEPARATION METHOD}

An earlier study [12] showed that complete independent solution of the equations in (7) is very complicated, and that the solution can be much facilitated by using additional information about the characteristics of the rain and ice medium. Paraboni and Mauri [10] solved the problem for a simplified case in which rain only causes differential attenuation and ice only causes differential phase shift (also included in the OPEX Reference Book on Depolarization [11], Section 2.5). However, this is not a realistic assumption, since it is known that rain also causes significant differential phase shift. They also suggested a more realistic (though still relatively simplified) case with a fixed ratio between differential attenuation and differential phase shift for rain, but in this case only the parameters for rain can be extracted from the expressions given; not those for ice.

In this paper, an improvement to the separation method from Paraboni and Mauri is presented, in which the parameters of both rain and ice can be calculated. For this purpose, a simulation is made of the parameters measured from a combination of rain and ice depolarization. In this simulation, the differential attenuation of the ice medium $\alpha_{i}$ will be assumed $0 \mathrm{~dB}$, and for the rain medium a certain relation between $\alpha_{r}$ and $\beta_{r}$ will be assumed (instead of a fixed ratio).

For the relation between $\alpha_{r}$ and $\beta_{r}$ of the rain medium, theoretical expressions have been published in literature by various researchers [13]-[19]. Because the calculation procedure will be tested on measurement results from Eindhoven, an expression must be used which most likely represents an average rainstorm in the Netherlands. It was found from an analysis of measured depolarization events [6] that the relation between $\alpha_{r}$ and $\beta_{r}$ depends on the type of rainstorm, due to variations of the drop size distribution (DSD). The average relation between $\alpha_{r}$ and $\beta_{r}$ therefore varies from event to event, but the relation always lies between the models from Fukuchi et al. [13], [14] for the Joss-thunderstorm DSD and for the Joss-drizzle DSD. The model by Ajayi [15], for drops of $10^{\circ} \mathrm{C}$, was found to be a good average relation. All these models use the drop shape model of Pruppacher-Pitter, which has an eccentricity and a flattened bottom both increasing with drop size, and a cavity at the bottom of the very largest drops.

For each of these three rain models, a relation of $\beta_{r}$ as a function of $\alpha_{r}$ was derived, valid for the dual-polarized Olympus link in Eindhoven, at a frequency of $19.77 \mathrm{GHz}$ and an elevation angle of $26.8^{\circ}$, and using the rain path length model from
ITU-R [20] and a spatial standard deviation of raindrop canting angles of $10^{\circ}$

$$
\begin{aligned}
& \beta_{r}=7.358 \alpha_{r}^{0.891}(\mathrm{deg}) \quad(\text { Ajayi }) \\
& \beta_{r}=5.920 \alpha_{r}^{0.744}(\mathrm{deg}) \quad(\text { Fukuchi }- \text { thunderstorm }) \\
& \beta_{r}=14.108 \alpha_{r}^{0.824}(\mathrm{deg}) \quad(\text { Fukuchi }- \text { drizzle })
\end{aligned}
$$

Equation (8) is a least-square-error fit to the model of Ajayi, with an error smaller than $0.3^{\circ}$ for $\alpha_{r}$ up to $8 \mathrm{~dB}$. Equation (9) and (10) are derived directly from the models of Fukuchi et al., which are valid for rain rates up to $50 \mathrm{~mm} / \mathrm{h}$, equivalent to $\alpha_{r} \leq 6.4 \mathrm{~dB}$ for (9) and $\alpha_{r} \leq 2.4 \mathrm{~dB}$ for (10).

In the simulation, $\alpha_{r}$ is taken as a characterizing parameter for rain, and $\beta_{r}$ is taken dependent from $\alpha_{r}$ according to any of (8)-(10). The depolarization ratios $X_{r}$ and $Y_{r}$ are calculated from these according to (4) and (3a), (3b), where the rainstorm canting angle $\phi_{r}$ is taken $0^{\circ}$ at this point, since the raindrops can be assumed to be on average horizontally orientated. This results in a range of complex values of $X_{r}$ and $Y_{r}$, with $\alpha_{r}$ as a characterizing parameter.

For the ice part, the differential attenuation $\alpha_{i}$ is taken 0 $\mathrm{dB}$, and the differential phase shift $\beta_{i}$ is the characterizing parameter. $X_{\mathrm{i}}$ and $Y_{\mathrm{i}}$ are also calculated equivalently to (4) and (3a), (b), where the ice cloud canting angle $\phi_{i}$ is taken equal to $\delta+45^{\circ}$, in order to maximize depolarization. This results in a range of complex values of $X_{\mathrm{i}}$ and $Y_{\mathrm{i}}$, with $\beta_{i}$ as a characterizing parameter. $\beta_{i}$ is given negative values as well. An ice cloud with a negative value of $\beta_{i}$ is equivalent to one with a positive $\beta_{i}$ and a canting angle $\phi_{i}$ rotated over $90^{\circ}$, so that this way two values of $\phi_{i}$ are already included in the simulation model.

It may be noted that this simulation neglects the contribution of the melting layer, in which ice crystals in the process of forming raindrops consist of a mixture of ice and water. These half-frozen hydrometeors are more random in shape than raindrops or ice crystals, but can still have a significant anisotropy. However, the error of neglecting it is not very large. Zhang, Tervonen, and Salonen [21] calculated the effect of forward scattering by melting layer particles. They found that the differential attenuation $\alpha$ and phase shift $\beta$ of these are smaller than those following from the rain models described above for the same frequencies and rain rates, while the relation between $\alpha$ and $\beta$ is roughly similar to the average relation for rain. It can be concluded that the depolarizing behavior of the melting layer is similar to that of rain. By neglecting its effect, it is effectively modeled as a small additional rain layer.

From $X_{\mathrm{r}}, Y_{\mathrm{r}}, X_{\mathrm{i}}$, and $Y_{\mathrm{i}}$, the simulated measured $X, Y$, and $R$ are calculated according to (7). Fig. 3 shows the parameter $X$ in the complex plane, for several constant $\beta_{i}$ values and increasing $\alpha_{r}$, and using Ajayi's rain model. In this figure, $X$ is imaginary for $\alpha_{r}=0 \mathrm{~dB}$ (when only the ice cloud is present), and moves over an approximate circle arch toward a point near 0 when $\alpha_{r}$ increases. When only the rainstorm is present, $X$ moves along an almost straight line for increasing $\alpha_{r}$.

As an auxiliary quantity, now the parameter $\Xi$ is introduced

$$
\Xi=\frac{1+R}{2 X}+\frac{1+\frac{1}{R}}{2 Y}
$$




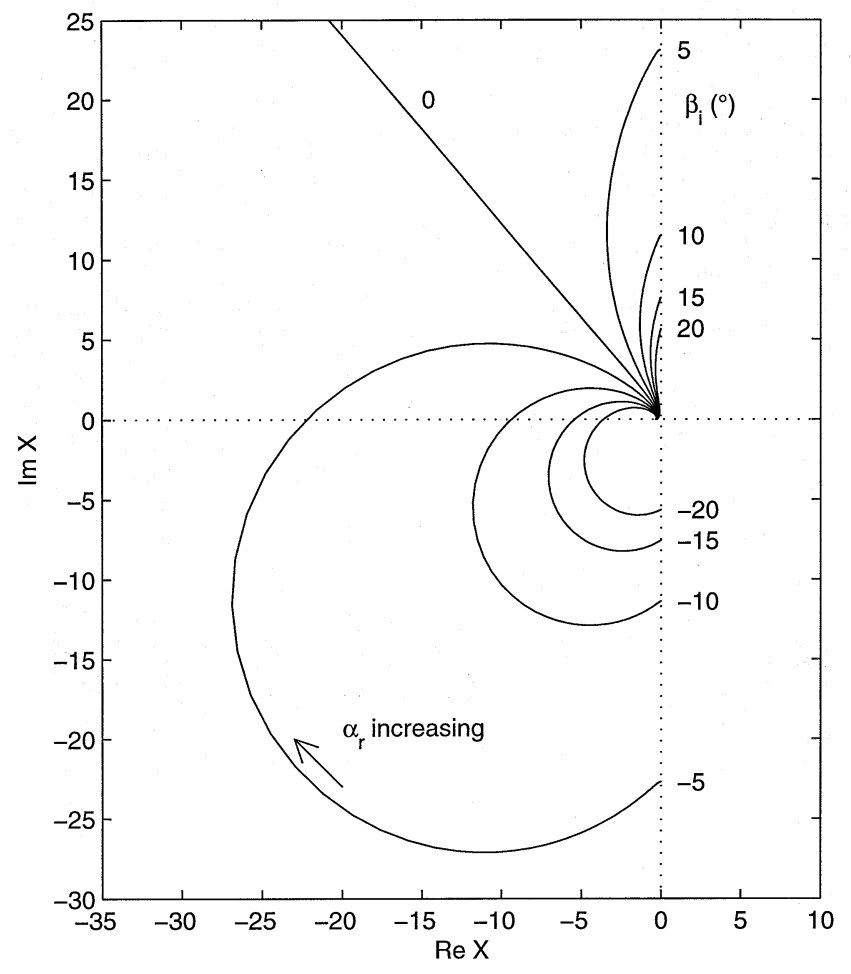

Fig. 3. $X$ in the complex plane, for increasing $\alpha_{r}$ from $0 \mathrm{~dB}$ upwards and for $\beta_{i}$ as indicated. Ajayi's rain model is used.

Fig. 4 shows $\Xi$ in the complex plane for rain depolarization using the three different rain depolarization models, and for ice depolarization. This figure shows that $\Xi$ is zero for clear sky, lies on the imaginary axis for ice depolarization, and lies on a curve in the third quadrant for rain depolarization. The position of this curve is dependent on the rain depolarization model. It can be seen that for a specific value of $\alpha_{r}$, all models (with different differential phase shifts) result in approximately the same real part of $\Xi$. This means that the real part of $\Xi$ is practically only influenced by differential attenuation. Similarly, it was also found (although not shown in this figure) that the imaginary part of $\Xi$ is practically only influenced by the differential phase shift of either ice or rain.

This result indicates that by observing $\Xi$ in the complex plane, it is possible to distinguish between differential attenuation effects and differential phase shift effects. Also, assuming one rain depolarization model, it will be possible to distinguish between rain, ice, and the combination of the two. In the following calculations, Ajayi's model is assumed.

Fig. 5 shows $\Xi$ in the complex plane, for a number of values of $\alpha_{r}$ and $\beta_{i}$, for depolarization due to a combination of rain and ice. This figure shows that, within the defined range, variations in $\alpha_{r}$ and $\beta_{i}$ cause almost mutually independent variations in $\Xi$. This means that even in the case of a combination of rain and ice, the real part of $\Xi$ is influenced only by differential attenuation. Similarly, the imaginary part is still only influenced by differential phase shift.

Approximate relations of $\alpha_{r}, \beta_{r}$ and $\beta_{i}$ with $\Xi$ are found as follows:

$$
\alpha_{r}=-14.50 \operatorname{Re} \Xi \mathrm{dB} \quad\left(\phi_{r}=0^{\circ}\right)
$$

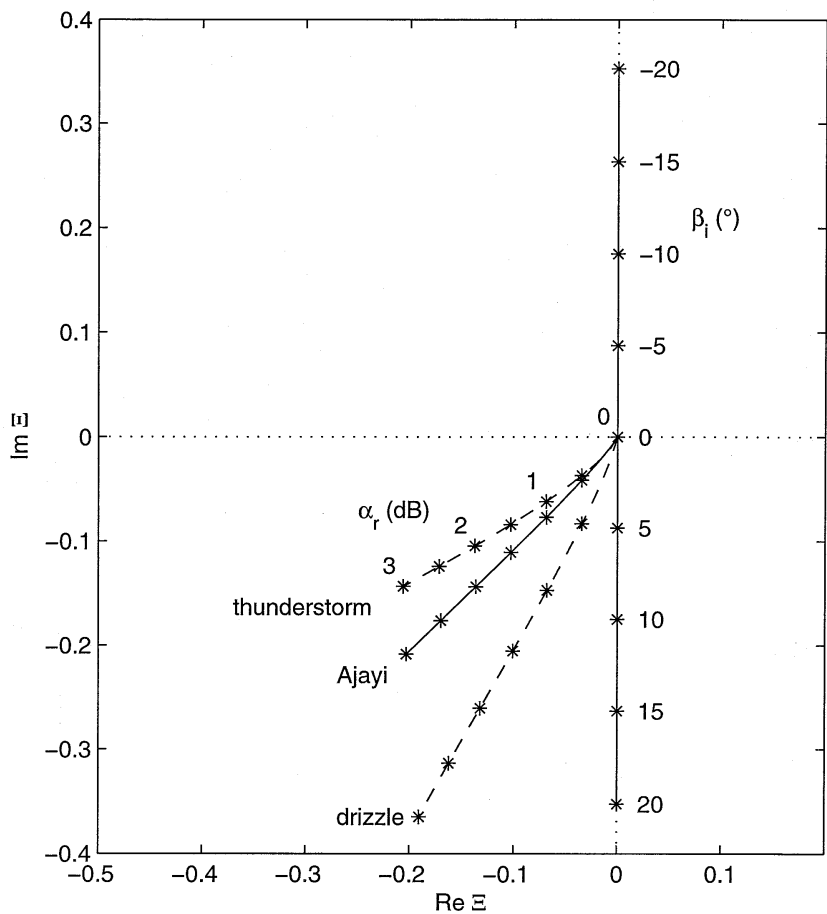

Fig. 4. $\Xi$ in the complex plane for rain depolarization $\left(\alpha_{r}=0 \ldots 3 \mathrm{~dB}\right.$; $\beta_{i}=0^{\circ}$ ), according to Ajayi's model and Fukuchi's thunderstorm- and drizzle-models, and for ice depolarization $\left(\beta_{i}=-20 \ldots+20^{\circ} ; \alpha_{r}=0 \mathrm{~dB}\right)$.

$$
\beta_{i}=-57.30 \operatorname{Im} \Xi-0.599 \beta_{r} \quad \operatorname{deg} \quad\left(\phi_{i}=\delta+45^{\circ}\right) .
$$

Using these equations, together with (8), $\alpha_{r}$ and $\beta_{i}$ can be extracted independently from a measurement of the complex quantity $\Xi$. The error of $\alpha_{r}$ calculated according to (12a), with respect to the input parameter $\alpha_{r}$ of the simulations, is in absolute value smaller than $0.12 \mathrm{~dB}$ for $0 \leq \alpha_{r} \leq 3 \mathrm{~dB}$ and $-20^{\circ} \leq \beta_{i} \leq+20^{\circ}$. The error of $\beta_{i}$ calculated according to (12b) and (8), with respect to the input parameter $\beta_{i}$, is in absolute value smaller than $1.0^{\circ}$ over the same range.

Fig. 5 is only valid under the assumptions mentioned before: the ice medium canting angle $\phi_{i}$ is $\delta+45^{\circ}$, and the rain canting angle $\phi_{r}$ is $0^{\circ}$. Next, the influence is evaluated of variations of both canting angles on the calculation result.

The parameter $\Xi$ of a simulated medium was calculated for $\alpha_{r}=0,1,2,3 \mathrm{~dB} ; \phi_{r}=0^{\circ}$; and $\beta_{i}=20^{\circ}$ (fixed), and the ice canting angle $\phi_{i}$ varying from $0^{\circ}$ to $180^{\circ}$ (which is the entire meaningful range for the canting angle, since $\phi_{i}=180^{\circ}$ is equivalent to $\phi_{i}=0^{\circ}$ ). The result is shown in Fig. 6. Comparing this figure with Fig. 5, it shows that when $\phi_{i}$ varies, $\Xi$ moves along approximately the same contours as when $\beta_{i}$ varies, especially for small values of $\alpha_{r}$. When varying the rain canting angle $\phi_{r}$, the situation appeared to be similar: $\Xi$ moves along approximately the same contour as when $\alpha_{r}$ varies.

Fig. 7 (left graph) shows the result of (12a) if it would be applied in the configuration with a varying rain canting angle. It appears that the result is approximately proportional to $\sin 2\left(\phi_{r}-\delta\right)$. Furthermore, it also appeared that the result of (12b) (not shown) is practically insensitive to $\phi_{r}$. When varying the ice canting angle $\phi_{i}$, the situation appeared to be the opposite: the result of $(12 b)$ is approximately proportional 


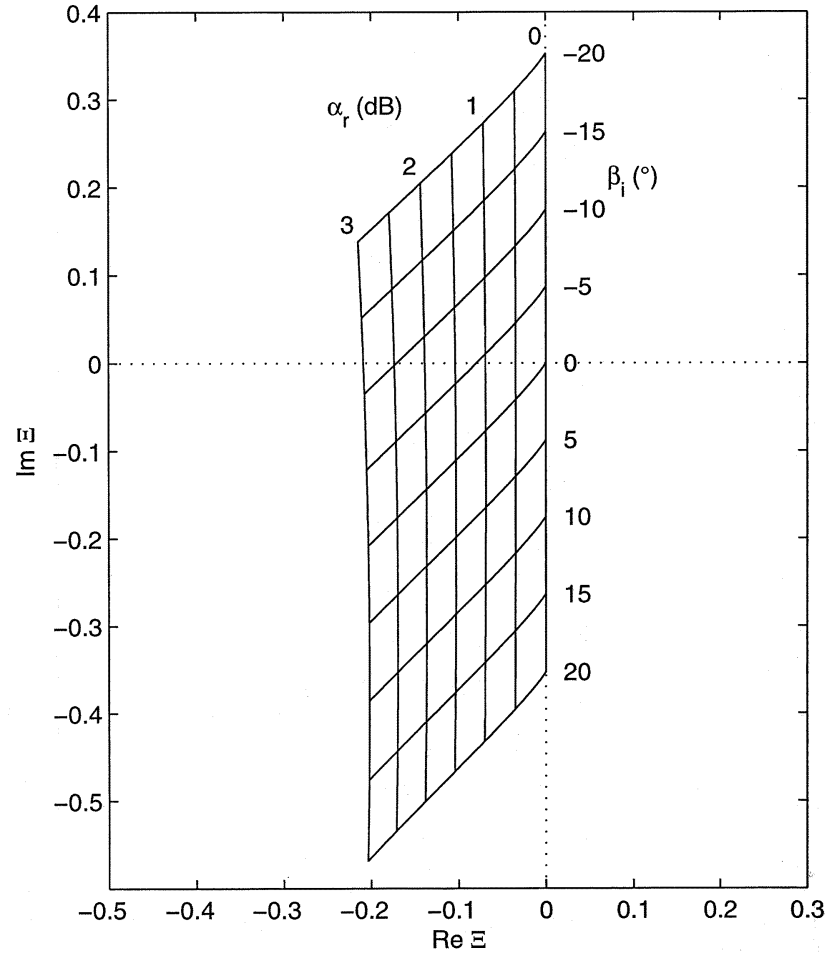

Fig. 5. $\Xi$ in the complex plane for polarization due to both rain and ice. The (almost) vertical lines are for constant $\alpha_{r}$ and variable $\beta_{i}$; the diagonal lines are for constant $\beta_{i}$ and variable $\alpha_{r}$.

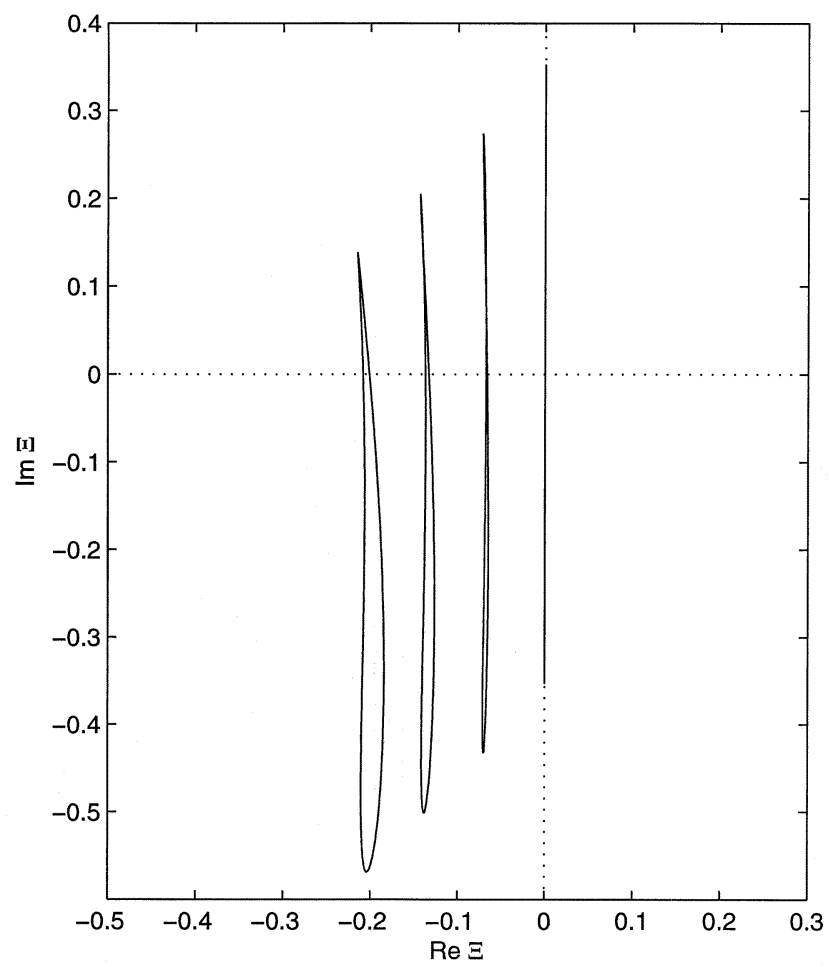

Fig. 6. $\Xi$ in the complex plane for $\alpha_{r}=0,1,2,3 \mathrm{~dB} ; \phi_{r}=0^{\circ} ; \beta_{i}=20^{\circ}$, and $\phi_{i}$ varying from 0 to $180^{\circ}$.

to $\sin 2\left(\phi_{i}-\delta\right)$, as shown in Fig. 7 (right graph), while the result of (12a) is hardly sensitive to $\phi_{i}$.
From these observations, it is found that the following approximations are valid for variable $\phi_{i}$ and $\phi_{r}$

$$
\begin{aligned}
& \alpha_{r} \sin 2\left(\phi_{r}-\delta\right)=-\frac{20}{\ln 10} \operatorname{Re} \Xi \mathrm{dB} \\
& \beta_{i} \sin 2\left(\phi_{i}-\delta\right)+\beta_{r} \sin 2\left(\phi_{r}-\delta\right)=-\frac{180}{\pi} \operatorname{Im} \Xi \mathrm{deg} .
\end{aligned}
$$

The canting angles $\phi_{r}$ and $\phi_{i}$ can also be calculated separately from the measurements, as is shown in the following. The quantities $\Xi$ and $R$ were calculated for $\alpha_{r}=1$ and $3 \mathrm{~dB}, \beta_{r}$ according to (8), $\beta_{i}=10$ and $20^{\circ}$, and $\phi_{r}$ and $\phi_{i}$ both varied from 0 to $180^{\circ}$. Fig. 8 shows that for these cases, the following approximations are valid.

$$
\begin{aligned}
\tan 2\left(\phi_{r}-\delta\right) & =\frac{\operatorname{Re} \Xi}{|R|-1} \\
\tan 2\left(\phi_{i}-\delta\right) & =\frac{\operatorname{Im} \Xi+\left(\frac{\pi}{180}\right) \beta_{r} \sin 2\left(\phi_{r}-\delta\right)}{\arg R+\left(\frac{\pi}{180}\right) \beta_{r} \cos 2\left(\phi_{r}-\delta\right)}
\end{aligned}
$$

$\phi_{r}$ and $\phi_{i}$ can be calculated from the measured quantities using (14a) and (14b), with the following additional clauses.

$$
\begin{array}{ll}
45^{\circ}<\phi_{r}-\delta<135^{\circ} & \text { if }|R|-1>0 \\
45^{\circ}<\phi_{i}-\delta<135^{\circ} & \text { if } \arg R+\left(\frac{\pi}{180}\right) \beta_{r} \cos 2\left(\phi_{r}-\delta\right)>0 .
\end{array}
$$

Using these formulas, all characterizing parameters of the rain and ice medium can be calculated from the measurements as follows. First, $\phi_{r}$ can be calculated from $\Xi$ and $R$ using (14a) and (15a). $\alpha_{r}$ can be calculated from $\Xi$ and $\phi_{r}$ using (13a). $\beta_{r}$ can be calculated from $\alpha_{r}$ using (8). Next, $\phi_{i}$ can be calculated from $\Xi, R, \beta_{r}$ and $\phi_{r}$ using (14b) and (15b). Finally, $\beta_{i}$ can be calculated from $\Xi, \beta_{r}, \phi_{r}$ and $\phi_{i}$ using (13b).

Fig. 9 shows the maximum errors in the calculated parameters using these formulas, in the ranges $0<\alpha_{r} \leq 2 \mathrm{~dB}, 0<\beta_{i} \leq$ $20^{\circ}, 0^{\circ}<\phi_{i} \leq 180^{\circ}$ and $0^{\circ}<\phi_{r} \leq 180^{\circ}$, as functions of $\phi_{r}$ and $\phi_{i}$. As this figure shows, the maximum error in $\alpha_{r}$ is 0.25 $\mathrm{dB}$, that in $\beta_{i}$ is $1.5^{\circ}$ and that in $\phi_{r}$ is $2.8^{\circ}$ in this range. The error in $\phi_{i}$ is only shown for $\beta_{i} \geq 10^{\circ}$, because the error becomes larger for smaller $\beta_{i}$. For $10 \leq \beta_{i} \leq 20^{\circ}$, the maximum error in $\phi_{i}$ is $4.5^{\circ}$.

It is not surprising that the error in $\phi_{i}$ becomes larger for smaller $\beta_{i}$, since it can be expected that the ice depolarization calculations will be distorted by the differential phase shift from rain when the ice depolarization component is relatively weak. In fact, in practice it can be expected that both canting angle calculations will be inaccurate whenever their respective source of depolarization is weak, since the canting angle is undefined when there is no anisotropy.

The described calculation procedure can be applied to any depolarization measurement from a dual polarized link. In measurement set-ups with a different frequency or elevation angle, or in climates where a different DSD can be expected, (8) should be replaced by an applicable expression of $\beta_{r}$ as a function of $\alpha_{r}$. These can be derived from values which have been published for various frequencies, elevation angles, and rain types 


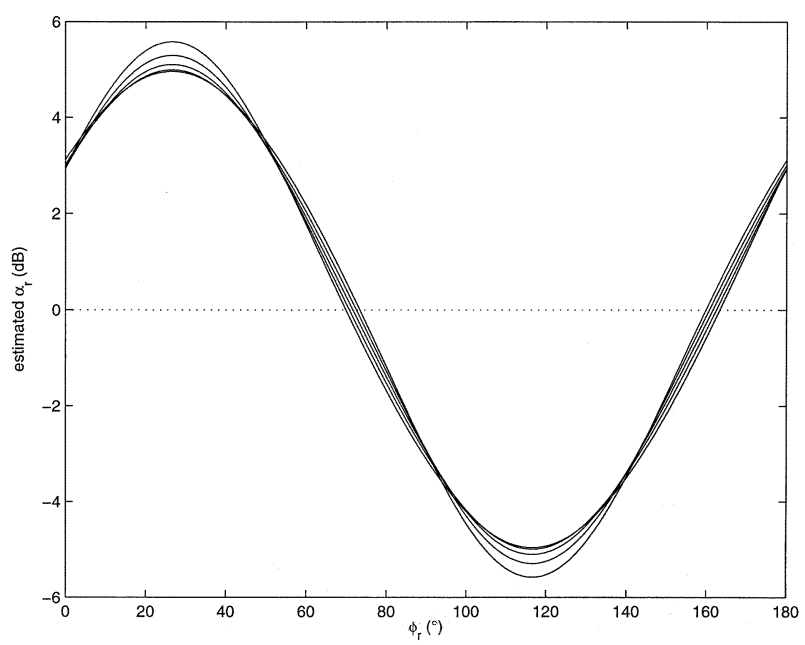

(a)

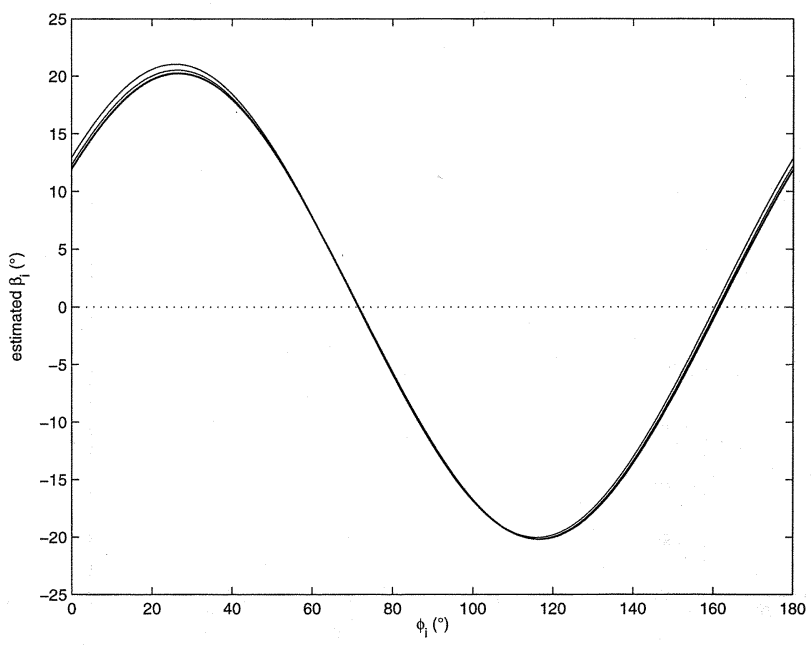

(b)

Fig. 7. (a) Result of (12a) when $\phi_{r}$ is varied; with $\alpha_{r}=3 \mathrm{~dB}, \phi_{i}=\delta+45^{\circ}, \beta_{i}=-20^{\circ},-10^{\circ}, 0^{\circ}, 10^{\circ}, 20^{\circ}$. (b) result of (12b) when $\phi_{i}$ is varied; with $\alpha_{r}=0,1,2,3 \mathrm{~dB}, \phi_{r}=0^{\circ}, \beta_{i}=20^{\circ}$.

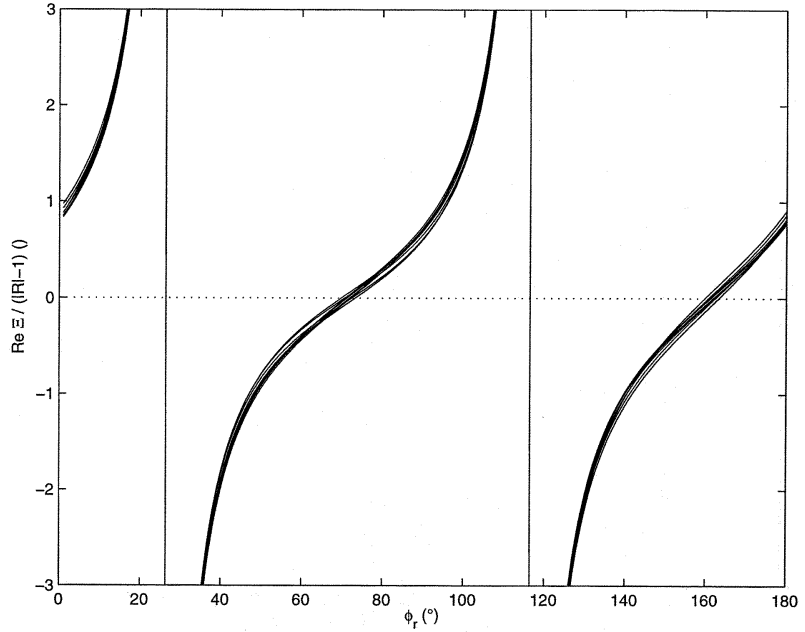

(a)

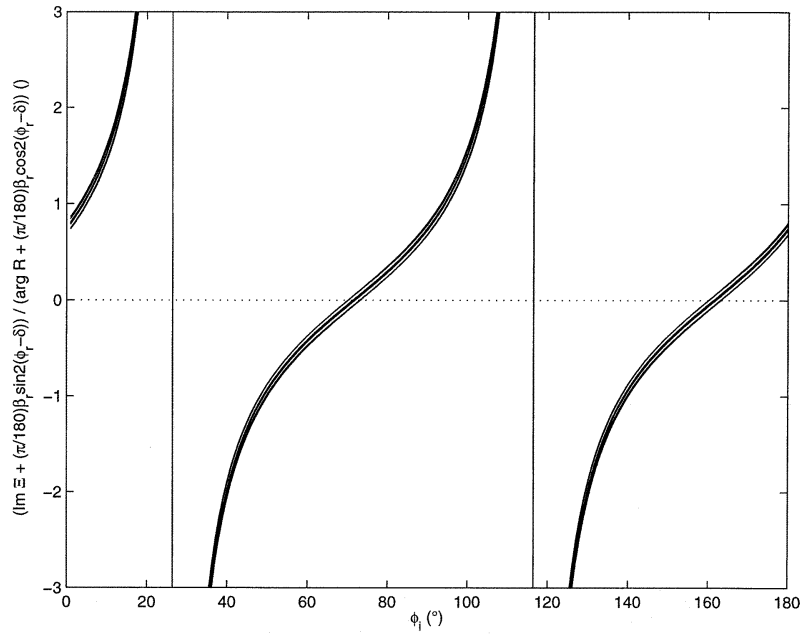

(b)

Fig. 8. (a) $\operatorname{Re} \Xi /(|R|-1)$ versus $\phi_{r}$ for various values of $\alpha_{r}, \beta_{i}$, and $\phi_{i}$. (b) $\left[\operatorname{Im} \Xi+(\pi / 180) \beta_{r} \sin 2\left(\phi_{r}-\delta\right)\right] /\left[\arg R+(\pi / 180) \beta_{r} \cos 2\left(\phi_{r}-\delta\right)\right]$ versus $\phi_{i}$ for various values of $\alpha_{r}, \beta_{i}$, and $\phi_{r}$. In both graphs, the different lines are very close together and hard to distinguish.

[13]-[19]. It should be noted that this expression need not be a power law, as (8)-(10); it can be any function. This provides another advantage of this separation method over the one from Paraboni and Mauri [10], [11] which used a fixed ratio between $\beta_{r}$ and $\alpha_{r}$. The advantage becomes especially large at higher frequencies (above $30 \mathrm{GHz}$ ), where the relation between $\beta_{r}$ and $\alpha_{r}$ deviates more and more from a straight line, and $\beta_{r}$ may even be negative for intense rainfall while positive for light rain, as can be seen from the tabulated values from Maggiori [17].

It was also studied which is the result of the calculation procedure in the case of a rainstorm which does not correspond to the rain type assumed in the calculation. In this test, $\Xi$ was calculated for a simulated rain medium, using Fukuchi's models for thunderstorm and drizzle-DSDs [13], [14] (which were seen to be two extreme cases for rainstorms in Eindhoven), and without ice. To these simulation results, (13)-(15) and (8) (assuming Ajayi's rain model) were applied. The relative error in the calculated $\alpha_{r}$ was found to be below $0.12 \mathrm{~dB}$ in both cases, for $\alpha_{r} \leq 2 \mathrm{~dB}$ and $\phi_{r}=0^{\circ}$. The calculated value of $\beta_{i}$, representing the amount of depolarization that is in these cases erroneously ascribed to ice, is shown in Fig. 10. This figure shows that, e.g., for $\alpha_{r}=0.5 \mathrm{~dB}$, the resulting $\beta_{i}$ can be up to $4^{\circ}$ in case of drizzle. This means that such resulting values of $\beta_{i}$ can be ignored as an indication of ice depolarization, but values of $\beta_{i}$ larger than given in Fig. 10 are a clear indication of the occurrence of ice depolarization.

The separation method described can be used to classify depolarization events as caused by rain, ice, or combined. For the combined events, $\alpha_{r}$ and $\beta_{i}$ can be calculated as indications of the contributions of depolarization from rain and from ice.

It is also possible to estimate from the measurements the XPD that would be measured from the separate rain and ice media alone, using (4), (3a), (3b), and (6a) and (6b). It is interesting to note that $X_{r}$ and $Y_{r}$ calculated this way are approximately proportional to $1 / \operatorname{Re} \Xi$. For a homogeneous medium, causing not too strong depolarization, $X$ and $Y$ 


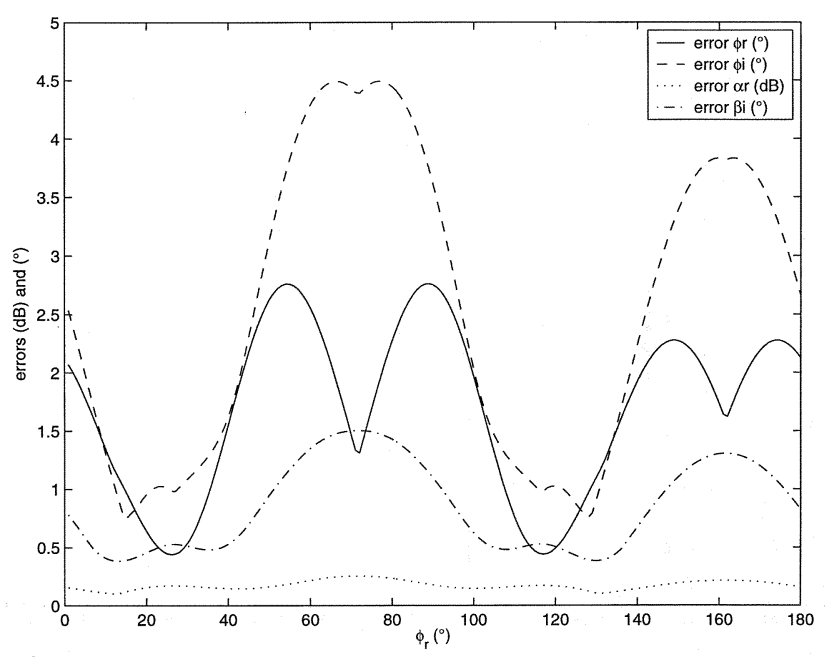

(a)

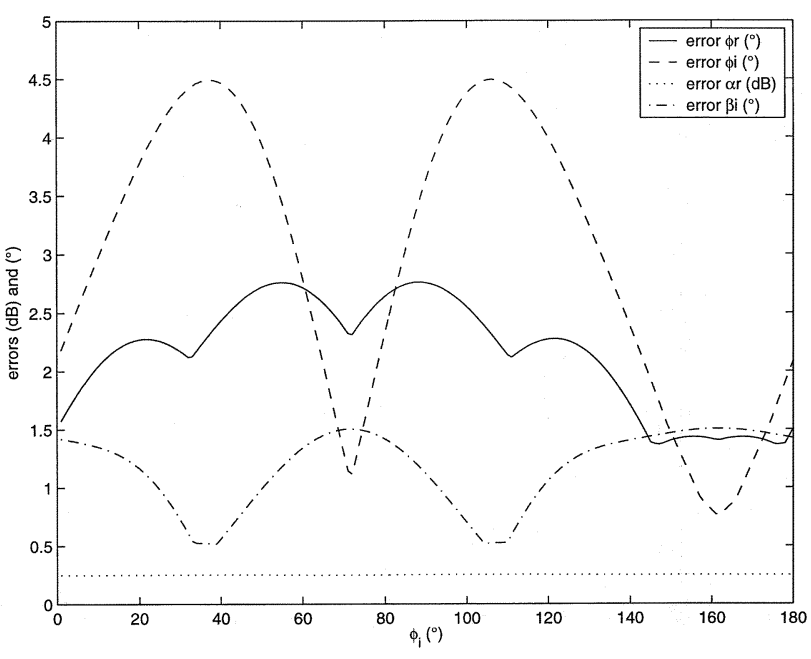

(b)

Fig. 9. (a) Maximum errors in calculated $\phi_{r}, \alpha_{r}, \phi_{i}$, and $\beta_{i}$ in the ranges $0<\alpha_{r} \leq 2 \mathrm{~dB}, 0<\phi_{i} \leq 180^{\circ}$ and $0<\beta_{i} \leq 20^{\circ}\left(\phi_{i}\right.$ only for $10 \leq \beta_{i} \leq 20^{\circ}$ ), as functions of $\phi_{r}$. (b) Maximum errors in calculated $\phi_{r}, \alpha_{r}, \phi_{i}$, and $\beta_{i}$ in the ranges $0<\alpha_{r} \leq 2 \mathrm{~dB}, 0<\phi_{r} \leq 180^{\circ}$ and $0<\beta_{i} \leq 20^{\circ}\left(\phi_{i}\right.$ only for $10 \leq \beta_{i} \leq 20^{\circ}$ ) as functions of $\phi_{i}$.

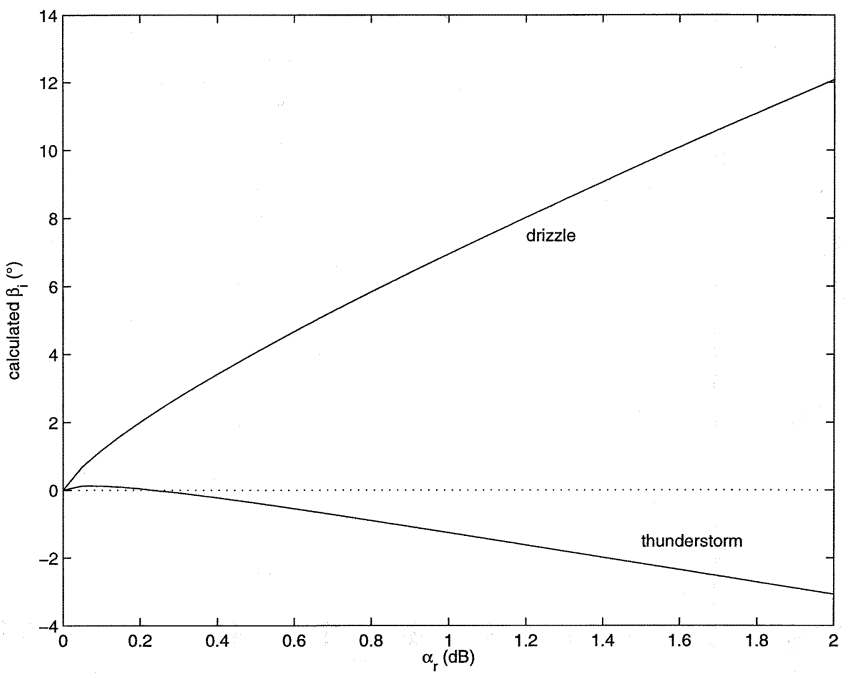

Fig. 10. Calculated $\beta_{i}$ in the case of a thunderstorm- or drizzle-type rainstorm, without ice, as a function of the calculated $\alpha_{r}$.

are approximately proportional to $\Delta$ and to $1 / \sin 2(\phi-\delta)$ [6]. Furthermore, according to the models $\beta_{r}$ is within a limited range approximately proportional to $\alpha_{r}$, so then also $\Delta_{r}=-\left(\alpha_{r}(\ln 10) / 20+\left(j \beta_{r} \pi / 180\right)\right)$ is proportional to $\alpha_{r}$. So, using (13a), $X_{\mathrm{r}}$ and $Y_{\mathrm{r}}$ of the rain medium are approximately proportional to

$$
\left.\begin{array}{c}
X_{r} \\
Y_{r}
\end{array}\right\} \propto \frac{1}{\Delta_{r} \sin 2\left(\phi_{r}-\delta\right)} \propto \frac{1}{\alpha_{r} \sin 2\left(\phi_{r}-\delta\right)} \propto \frac{1}{\operatorname{Re} \Xi} .
$$

This means that the vertical lines in Fig. 5 are contours indicating XPD that would be measured from the rain part alone. In a similar way it can be shown that $X_{i}$ and $Y_{i}$ are approximately proportional to $1 / \beta_{i} \sin 2\left(\phi_{i}-\delta\right)$, so that the diagonal lines in
Fig. 5 are contours of XPD due to ice. Therefore, the measurement and plotting of the complex quantity $\Xi$ gives immediately an insight in the contributions of both sources of depolarization. This elegant property of this separation method is yet another improvement with respect to the method from Paraboni and Mauri.

\section{ExPerimental Test of SeParation Method}

In this section, the calculation method described in the previous section is tested on several measured depolarization events.

The dual-polarized $19.77 \mathrm{GHz}$ beacon from the satellite Olympus was measured at Eindhoven University of Technology using a $5.5 \mathrm{~m}$ Cassegrain antenna. The local polarization angle was $71.6^{\circ}$, and the elevation angle was $26.8^{\circ}$. XPD, $\psi$, and CoPolar Attenuation $(C P A)$ at both polarizations were sampled at a frequency of $3 \mathrm{~Hz}$ and averaged over every second.

In the graphs of Fig. 11, the plots of the measured XPD and $C P A$ at $y$-polarization are shown for a rain event, an ice event and a combined event. The typical characteristics of the different events can be seen from these: the rain event shows strong depolarization and strong attenuation, the ice event shows strong depolarization and practically no attenuation, and the combined event shows strong depolarization which only partly coincides with strong attenuation. In this last case is it likely that during the gradual increase of attenuation, rain and ice simultaneously cause depolarization on the propagation path.

In Fig. 12, the parameter $\Xi$ as defined in (11) is shown in a scatterplot for each of the three events. The typical behavior of the model of the previous section is clearly observed for the first two events: During the rain event, $\Xi$ is in the third quadrant, around the curve following from Ajayi's model. During the ice event, $\Xi$ stays around the imaginary axis. For the combined event, $\Xi$ shows two main peaks: one on the imaginary axis and 


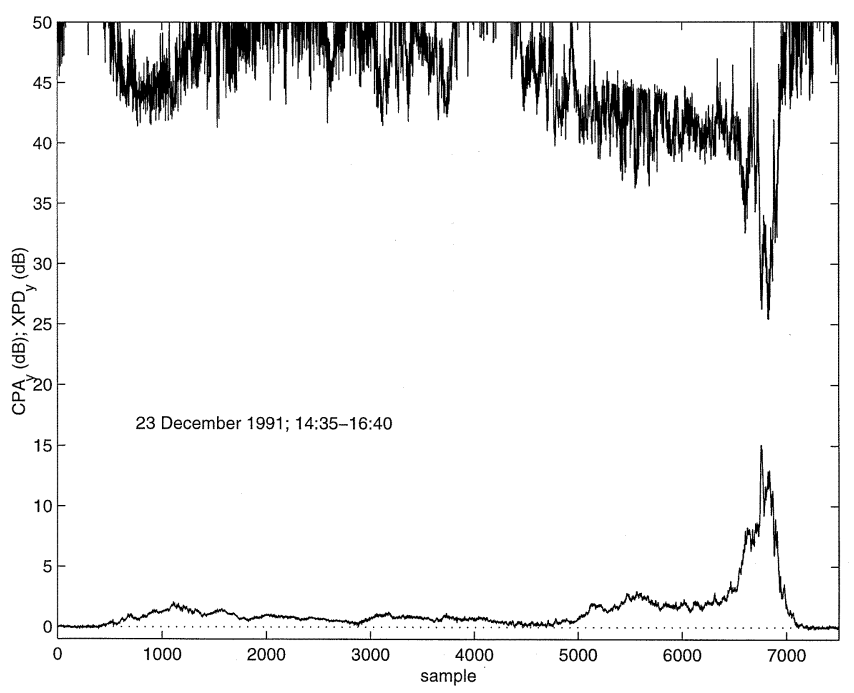

(a)

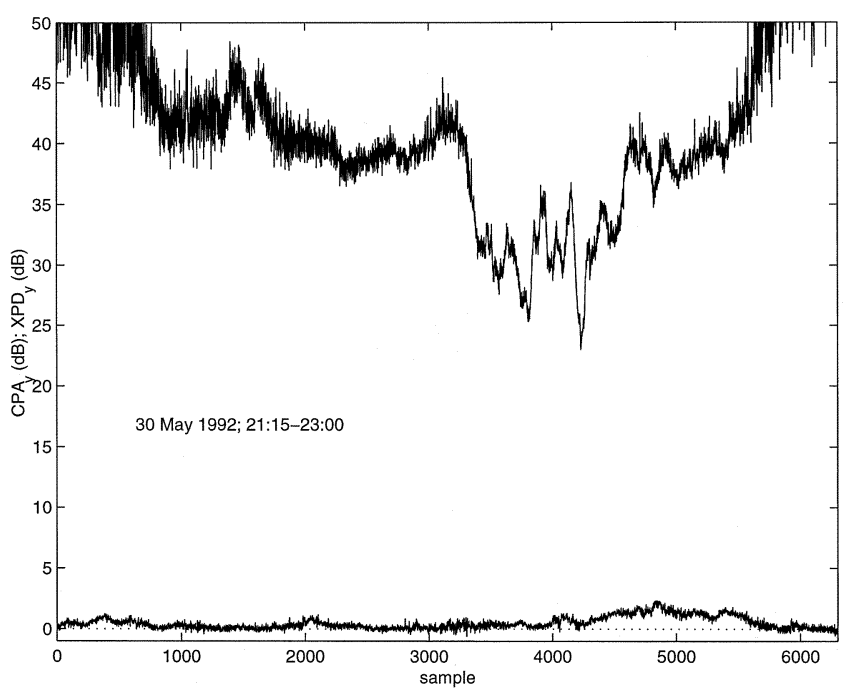

(b)

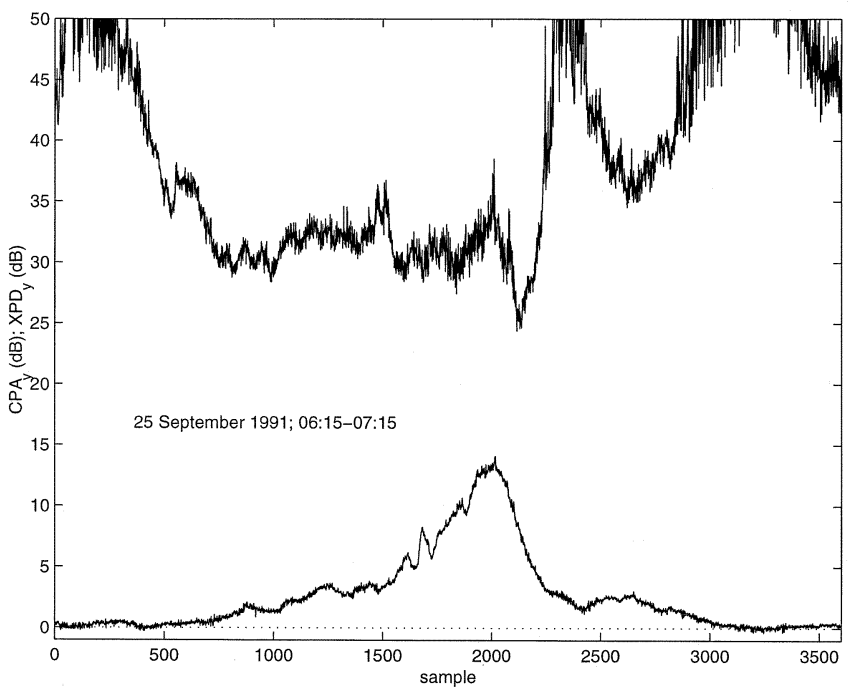

(c)

Fig. 11. XPD (upper curve) and $C P A$ at $y$ polarization, measured for (a) a rain event, (b) an ice event, and (c) a combined event.

one on the rainy curve. Outside of those peaks, $\Xi$ moves between these two curves. It is, therefore, expected that the calculation procedure of the previous section will be able to detect the variable contributions of rain and ice depolarization.

Fig. 13 shows the calculated results for $\alpha_{r}, \beta_{i}, \phi_{r}$, and $\phi_{i}$. This figure shows that the procedure is well able to recognize the different kinds of events: in the first event, all depolarization is ascribed to rain, in the second, to ice, and in the third, to both. Especially from sample 1600 to sample 1900 in this last event, both rain and ice appear to contribute significantly to the depolarization. The two main peaks during the combined part of this event are also clearly recognized: for rain it occurs at sample 1985 and for ice at sample 2133.

The two canting angles appear to show relatively clear values when their respective source of depolarization is present. Not surprisingly, the calculation of $\phi_{r}$ is unstable when $\alpha_{r} \leq 0.2 \mathrm{~dB}$ and that of $\phi_{i}$ is unstable when $\beta_{i} \leq 1^{\circ}$; under those conditions, the calculated canting angles show large jumps and fluctuations. This was expected, since the canting angle is undefined when there is no anisotropy. It appears that during significant depolarization $\left(\alpha_{r}>0.2 \mathrm{~dB}\right.$ or $\left.\beta_{i}>1^{\circ}\right)$, both canting angles tend to be stable at values slightly below $0^{\circ}$. Only these values should be regarded as true results of the actual rain and ice canting angles.

In the case of a different kind of rainstorm than corresponding to the model, the calculated value of $\beta_{i}$, representing the amount of depolarization that is in this case erroneously ascribed to ice, was shown in Fig. 10. In Fig. 13, it can be seen that for the rain event [Fig. 13(a)-(d)], $\beta_{i}$ stays below these values, which means that such resulting values of $\beta_{i}$ can be ignored as an indication of ice depolarization. For the combined event [Fig. 13(i)-(1)], however, $\beta_{i}$ exceeds these values, which indicates clearly the occurrence of ice depolarization.

\section{CONCLUSION}

Simultaneous depolarization by rain and ice crystals can be separated effectively using the method described in this paper. 


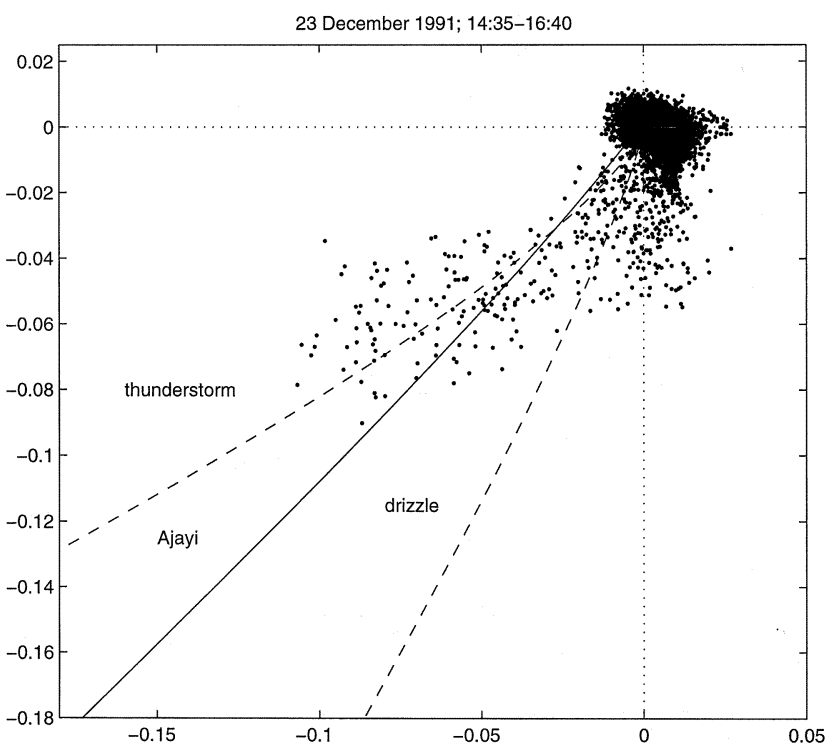

(a)

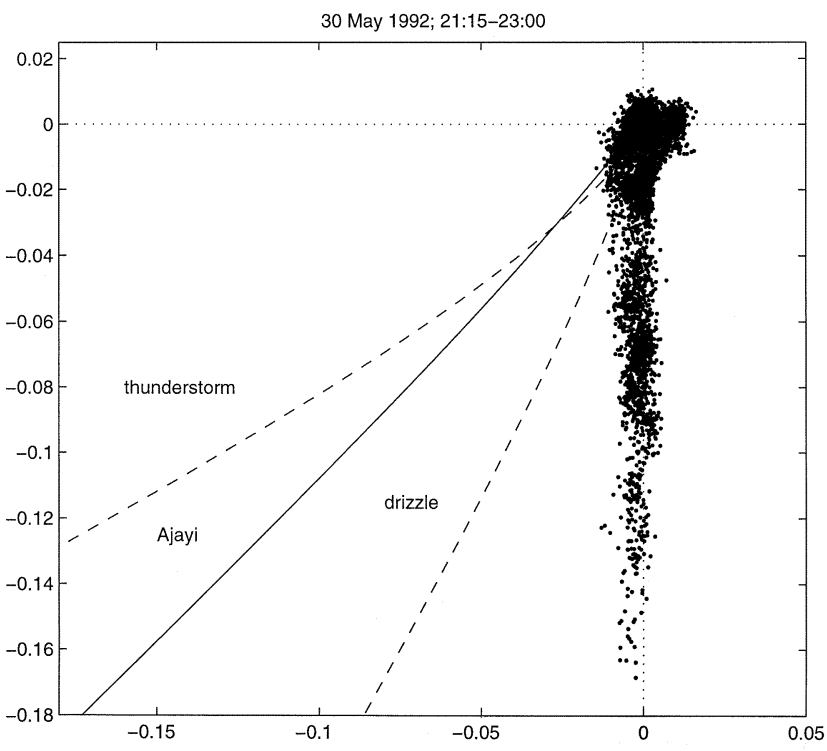

(b)

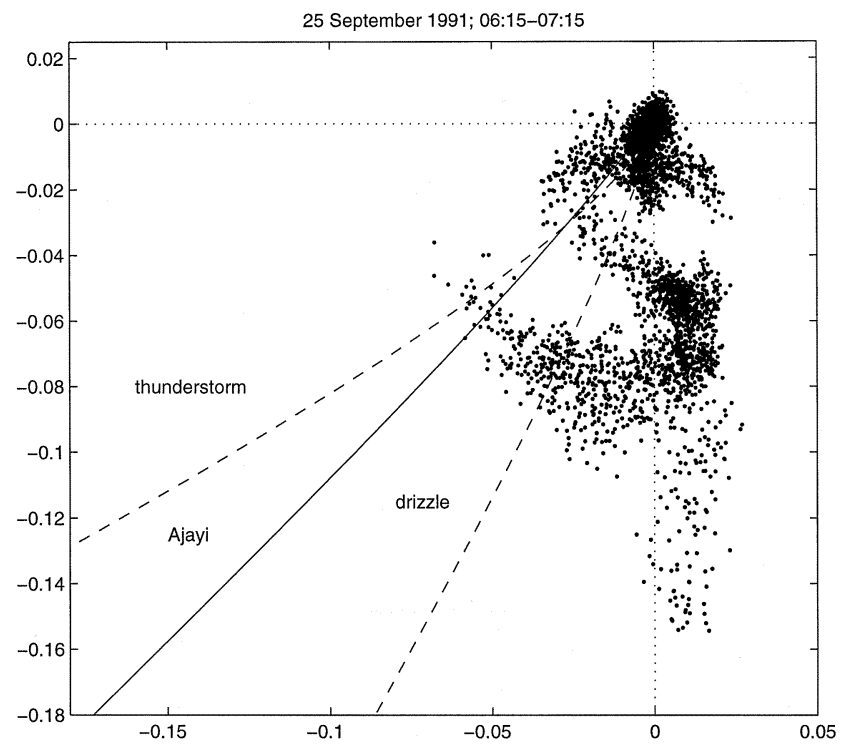

(c)

Fig. 12. $\Xi$ in the complex plane for the three events of Fig. 11: (a) rain, (b) ice, and (c) combined. Also included are the curves for rain depolarization according to the three rain models.

The separate contributions of rain and ice are clearly distinguished by plotting the complex parameter $\Xi$. This method can be used to classify events as rain, ice or combined events. For the combined events, the characteristics parameters of rain and ice can be calculated from the measurements using (13)-(15) and (8). For measurements at other frequencies or elevation angles or in different climates, (8) should be replaced by an applicable expression.

This tool can help to generate statistics of the characteristic parameters of rain and ice depolarization, which in turn can lead to improved prediction models of these depolarization effects. Also, XPD that would be measured from propagation through the separate media alone can be calculated, so that statistics thereof can be derived.
The separation method presented has several improvements to the method from Paraboni and Mauri:

- it is possible to calculate all parameters of rain and ice separately;

- it can be easily adapted to any other relation between differential attenuation and differential phase shift of rain;

- it shows the different contributions of depolarization immediately and clearly in one complex parameter.

Apart from its direct application to propagation modeling and system design, this technique might also improve the applicability of propagation measurements for remote sensing of the troposphere. Using an array of spatially separated dual polarized links, an image of the horizontal structure of rain storms 


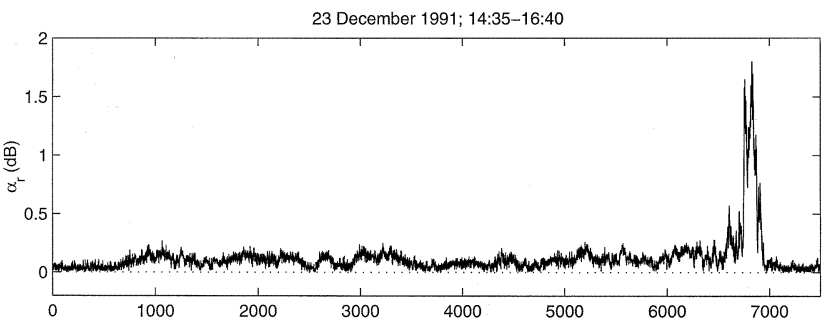

(a)

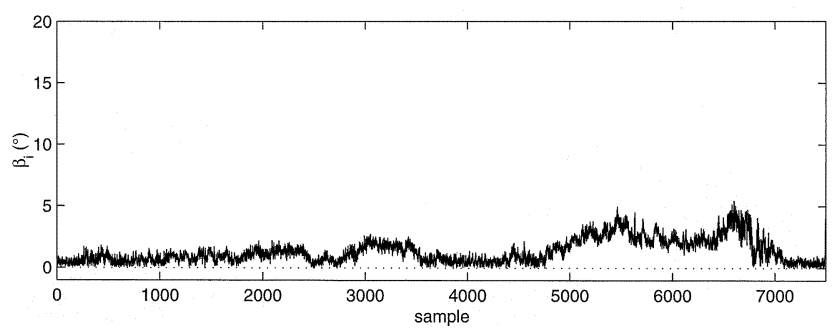

(c)

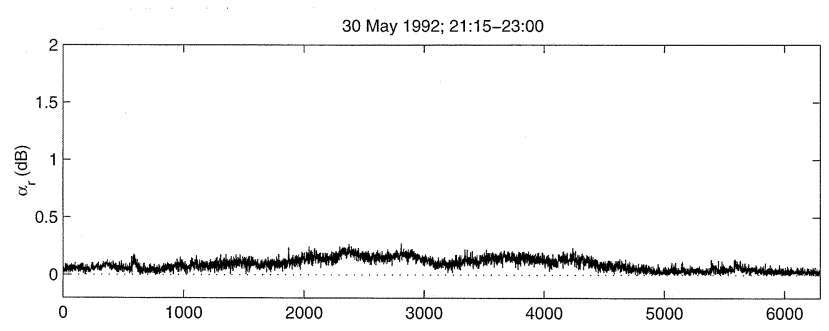

(e)

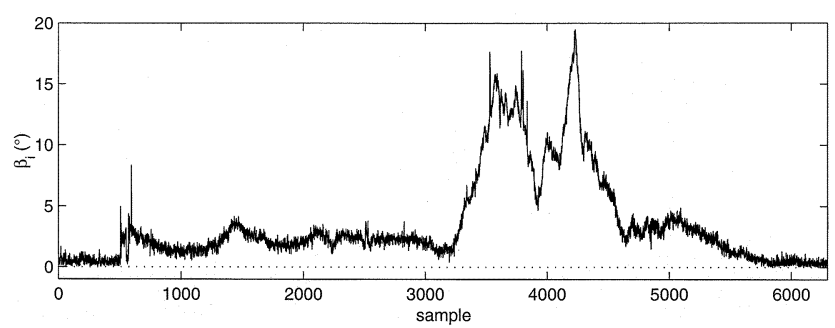

(g)

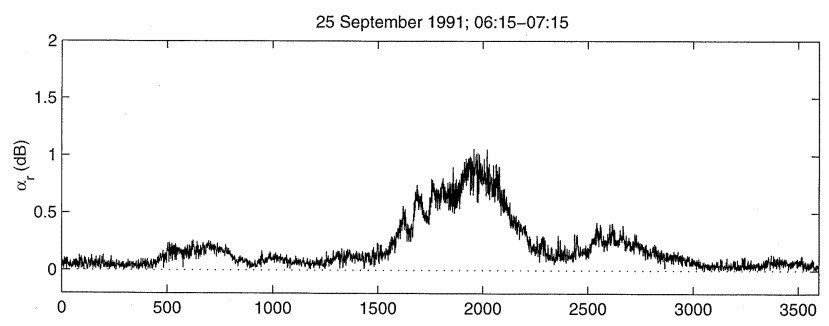

(i)

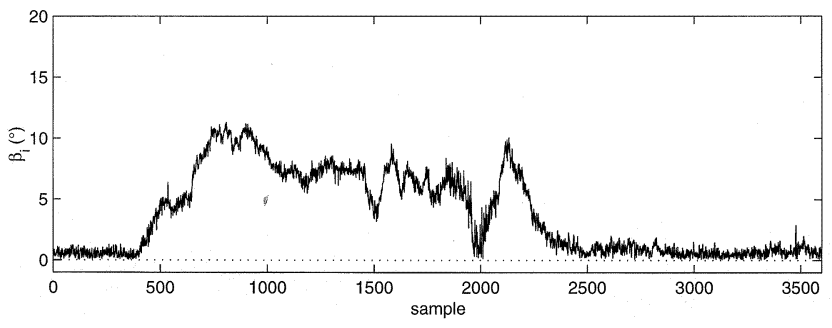

(k)

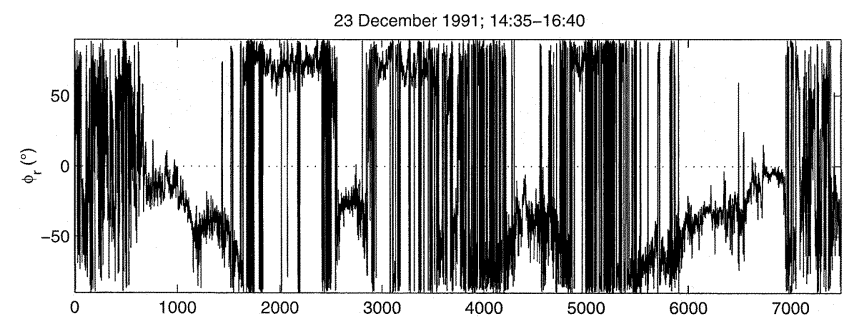

(b)

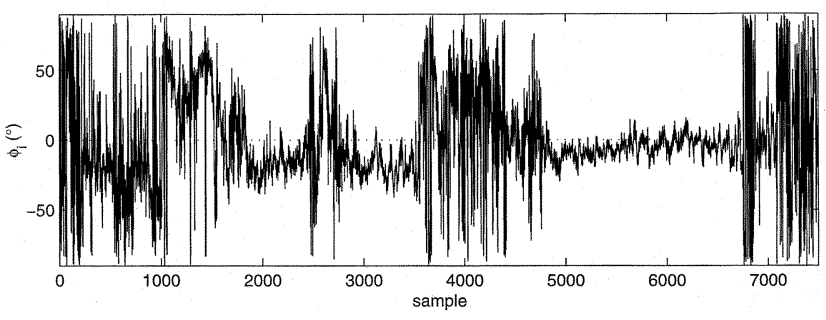

(d)

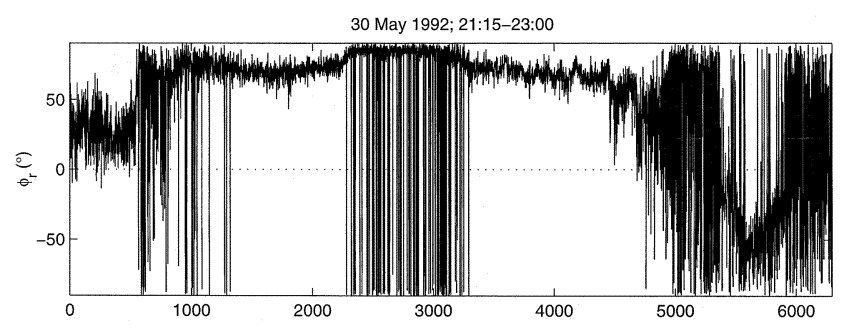

(f)

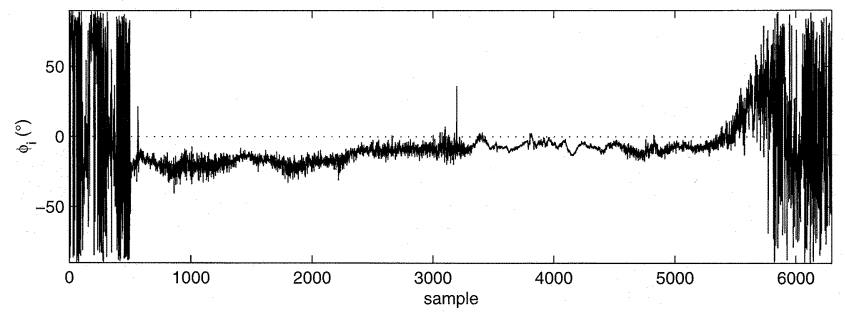

(h)

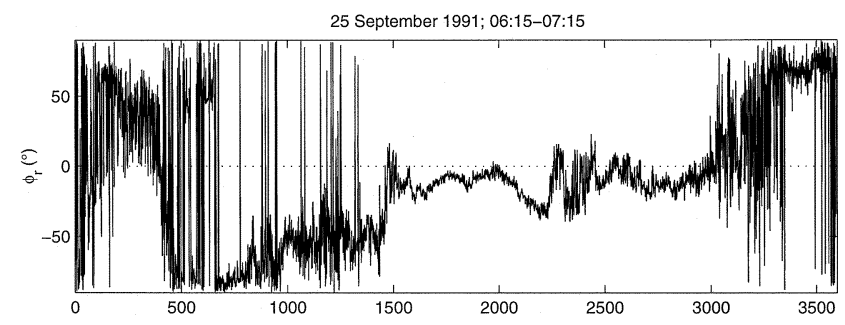

(j)

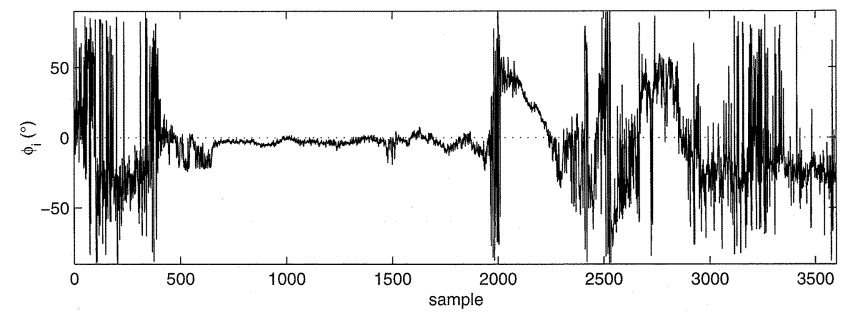

(1)

Fig. 13. (a), (e), and (i) $\alpha_{r}$; (b), (f), (j) $\phi_{r}$; (c), (g), and (k) $\beta_{i}$; and (d), (h), and (1) $\phi_{i}$, calculated from the three events of Fig. 11: (a)-(d) rain, (e)-(h) ice, and (i)-(l) combined. Canting angles above $90^{\circ}$ have been plotted as negative angles. 
and ice clouds may be obtained. However, radar measurements will still be necessary if information is needed on the vertical structure.

\section{REFERENCES}

[1] G. Brussaard, "A meteorological model for rain-induced cross polarization," IEEE Trans. Antennas Propagat., vol. AP-24, pp. 5-11, Jan. 1976.

[2] G. Brussaard and P. A. Watson, Atmospheric Modeling and Millimeter Wave Propagation. London: Chapman and Hall, 1995.

[3] H. Fukuchi, J. Awaka, and T. Oguchi, "Improved theoretical formula for the relationship between rain attenuation and depolarization," Electron. Lett., vol. 20, no. 21, pp. 859-860, 1984.

[4] R. A. Hogers, M. H. A. J. Herben, and G. Brussaard, "Distinction between rain and ice depolarization by calculation of differential attenuation and phase shift," Electron. Lett., vol. 27, no. 19, pp. 1752-1753, 1991.

[5] A. Aresu, A. Martellucci, and A. Paraboni, "Experimental assessment of rain anisotropy and canting angle in a horizontal path at $30 \mathrm{GHz}, "$ IEEE Trans. Antennas Propagat., vol. 41, pp. 1331-1335, 1993.

[6] M. M. J. L. van de Kamp, "Climatic radiowave propagation models for the design of satellite communication systems," Ph.D. dissertation, Eindhoven Univ. Technol., Nov. 1999.

[7] D. P. Haworth, P. A. Watson, and N. J. McEwan, "Model for the effect of electric fields on satellite-earth microwave radio propagation," Electron. Lett., vol. 13, no. 19, pp. 562-564, 1977.

[8] P. Golé and J. P. Mon, "Effect of ice-induced cross-polarization on digital earth-space links," IEEE Trans. Commun., vol. COM-32, pp. 70-76, Jan. 1984.

[9] F. Dintelmann, G. Ortgies, F. Rücker, and R. Jakoby, "Results from 12to $30-\mathrm{GHz}$ German propagation experiments carried out with radiometers and the Olympus satellite," Proc. IEEE, vol. 81, no. 6, pp. 876-884, 1993.

[10] A. Paraboni and M. Mauri, "The physical basis of depolarization," in Proc. Olympus Utilization Conf., Sevilla, Spain, 1993, pp. 573-581.

[11] F. Dintelmann, Ed., "OPEX; volume 2: reference book on depolarization," in Second Workshop of the OLYMPUS Propagation Experimenters. Noordwijk, The Netherlands: ESA/ESTEC, 1994.

[12] M. M. J. L. van de Kamp, "Dual-Channel Crosspolarization Analysis," Final Report of ESA/ESTEC Contract 10290/93/NL/GS, Eindhoven Univ. Tech., 1994.

[13] H. Fukuchi, J. Awaka, and T. Oguchi, "Frequency scaling of depolarization at centimeter and millimeter waves," Electron. Lett., vol. 21, no. 1, pp. 10-11, 1985.
[14] _ - "A theoretical formula for the prediction of cross-polarized signal phase," IEEE Trans. Antennas Propagat., vol. AP-33, pp. 997-1002, Sept. 1985

[15] G. O. Ajayi, "Characteristics of rain-induced attenuation and phase shift at $\mathrm{cm}$ and $\mathrm{mm}$ waves using a tropical raindrop size distribution model," Int. J. Infrared and Millim. Waves, vol. 6, pp. 771-806, 1985.

[16] D. J. Fang and C. H. Chen, "Propagation of centimeter/millimeter waves along a slant path through precipitation," Radio Sci., vol. 17, no. 5, pp. 989-1005, 1982.

[17] D. Maggiori, "Computed transmission through rain in the 1-400 GHz frequency range for spherical and elliptical drops and any polarization," Alta Frequenza, vol. 50, no. 5, pp. 262-273, 1981.

[18] T. Oguchi, "Scattering properties of Pruppacher-and-Pitter form raindrops and cross polarization due to rain: calculations at 11, 13, 19.3, and $34.8 \mathrm{GHz}$," Radio Sci., vol. 12, no. 1, pp. 41-51, 1977.

[19] ITU-R, Recommendations of the ITU-R, p. 838, 2001.

[20] - Recommendations of the ITU-R, p. 839, 2001

[21] W. Zhang, J. K. Tervonen, and E. T. Salonen, "Backward and forward scattering by the melting layer composed of spheroidal hydrometeors at 5-100 GHz," IEEE Trans. Antennas Propagat., vol. 44, pp. 1208-1219, 1996.

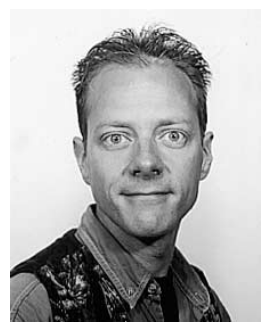

Max M. J. L. van de Kamp was born in Driebergen, the Netherlands, in 1963. He received the M.Sc. degree in electrical engineering and the Ph.D. degree in telecommunications from Eindhoven University of Technology (EUT), The Netherlands, in 1989 and 1999 , respectively.

He has been working as a Research Scientist in different radiowave propagation research projects: during 1990 to 1994 at EUT; during 1995 to 1997 at Helsinki University of Technology, Finland; during 1997 to 1999 again at EUT; and from 2000 to 2003, with Onera, Toulouse, France; and since 2003, with the Department of Electrical and Electronic Engineering, University of Bath, Bath, U.K. His activities in these projects have been studies of rain and ice depolarization, tropospheric scintillation, and dynamic aspects of rain attenuation. During his various research jobs, he has been involved in international cooperational satellite propagation research projects of the European Space Agency: the Olympus Propagation Experiment (OPEX) from 1991 to 1994, COST255 from 1995 to 1999, and COST280, since 2001. 